
gular systems of differential equations," SIAM J. Alg. Discrete Meth., vol. 4, pp. 517-521, 1983.

[8] I. M. Gelf́and and G. E. Shilov, Generalized Functions. Beijing, China: Science Press, 1965, vol. I.

[9] L. Dai, Singular Control Systems. Berlin, Germany: Springer-Verlag, 1989.

[10] A. I. G. Vardulakis, Linear Multivariable Control-Algebraic Analysis and Synthesis Methods. New York: Wiley, 1991. tems," IEEE Trans. Autom. Control, vol. 50, no. 11, pp. 1796-1799, Nov. 2005.

[12] K. J. Åström, Introduction to Stochastic Control Theory. New York: Academic, 1970.

[13] J. Y. Ishihara and M. H. Terra, "Impulse controllability and observability of rectangular descriptor systems," IEEE Trans. Autom. Control, vol. 46, no. 6, pp. 991-994, Jun. 2001.

\section{Robust Partial Pole Assignment for Vibrating Systems With Aerodynamic Effects}

\author{
Biswa N. Datta, Wen-Wei Lin, and Jenn-Nan Wang
}

[7] S. L. Campbell and L. R. Petzold, "Canonical forms and solvable sin-

[11] Z. Yan and G. Duan, "Time domain solution to descriptor variable sys-

where $M$ is the inertia matrix and $C_{1}, K_{1}, C_{2}$, and $K_{2}$ are the structural damping, structural stiffness, aerodynamic damping, and aerodynamic stiffness matrices, respectively. The nonhomogeneous term $H(s, t)$ represents the forcing function which is the combination of the generalized forces and gust inputs. Here, the parameter $s$ is interpreted as the Laplace transform parameter. For more rigorous interpretation of (1), we refer to [28]. Roughly speaking, the terms involving parameter $s$ should be interpreted as convolution operations. In most practical applications, the matrices $M, K_{1}, K_{2}$ are real positive definite and $C_{1}, C_{2}$ are real symmetric. However, we assume throughout the note that $M, C_{1}, C_{2}, K_{1}, K_{2}$ are real symmetric and $M$ is nonsingular. The factor $\zeta(s)$ in (1) is called the Wagner lift-growth buildup function which is due to an instantaneous change in angle of attack [14]. In this work, we take

$$
\zeta(s)=\rho+\frac{\rho}{s-\omega}
$$

with constants $\rho \neq 0$ and $\omega \neq 0$. We first consider the homogeneous case where $H(s, t) \equiv 0$ in (1). Multiplying $s-\omega$ on both side of (1), treating $s$ as the $t$ derivative, and setting $q=x e^{\lambda t}$ with $x \in \mathbb{C}^{n}$, the system (1) leads to a cubic open-loop matrix polynomial (see [12] and [20]):

$$
P(\lambda)=M \lambda^{3}+C \lambda^{2}+K \lambda+L
$$

where

$$
\begin{aligned}
C & =C_{1}+\rho C_{2}-\omega M \\
K & =\left(K_{1}+\rho K_{2}\right)-\omega\left(C_{1}+\rho C_{2}\right)+\rho C_{2} \\
L & =\rho K_{2}-\omega\left(K_{1}+\rho K_{2}\right) .
\end{aligned}
$$

Now choosing the control force $H(s, t)=B F^{T} \dot{q}+B\left(G_{1}^{T}+\right.$ $\left.\zeta(s) G_{2 T}\right) q$ in (1), we obtain a controlled system, which gives rise to a closed-loop matrix polynomial [7]:

$$
\begin{aligned}
P_{c}(\lambda)=M \lambda^{3} & +\left(C-B F^{T}\right) \lambda^{2}+\left(K-B G_{1}^{T}-\rho B G_{2}^{T}\right. \\
& \left.+\omega B F^{T}\right) \lambda+\left(L-\rho B G_{2}^{T}+\omega B G_{1}^{T}+\omega \rho B G_{2}^{T}\right) .
\end{aligned}
$$

Index Terms-Cubic matrix ploynomial, least-squares problem, robust partial ploe, vibrating systems.

\section{INTRODUCTION}

Consider the following general model of vibrating systems with aerodynamics effects:

$$
M \ddot{q}+\left(C_{1}+\zeta(s) C_{2}\right) \dot{q}+\left(K_{1}+\zeta(s) K_{2}\right) q=H(s, t)
$$

Manuscript received September 10, 2004; revised October 6, 2005 and April 27, 2006. Partially supported by NSF Grant DMS- 0505784 .

This note first appeared in the Proceedings of the 43rd IEEE Conference on Decision and Control, Bahamas, December 2004.

B. N. Datta is with the Department of Mathematical Sciences, Northern Illinois University, Dekalb, IL 60115 USA (e-mail: dattab@math.niu.edu).

W.-W. Lin is with the Department of Mathematics, National Tsing-Hua University, Hsinchu 300, Taiwan.

J.-N. Wang is with the Department of Mathematics, National Taiwan University, Taipei 106, Taiwan.

Digital Object Identifier 10.1109/TAC.2006.886543
Here, $B \in \mathbb{R}^{n \times b}$ is the control matrix and $F, G_{1}, G_{2} \in \mathbb{R}^{n \times b}$ are the assume throughout that $B$ has full-column rank. Let $\left\{\lambda_{j}\right\}_{j=1}^{3 n}$ be the self-conjugate spectrum of $P(\lambda)$ and $\left\{\mu_{j}\right\}_{j=1}^{k}$ with $1 \leq k<3 n$ be another self-conjugate set. Then the partial pole assignment problem for the above cubic pencil is to find real gain matrices $F, G_{1}, G_{2}$ such that $\left\{\left\{\mu_{j}\right\}_{j=1}^{k},\left\{\lambda_{j}\right\}_{j=k+1}^{3 n}\right\}=\sigma\left(P_{c}\right)$. Here and after, $\sigma(Q)$ denotes the spectrum of the matrix polynomial $Q(\lambda)$ or the spectrum of the matrix $Q$. In other words, one would like to use the low rank perturbations $B F^{T}, B G_{1}^{T}$, and $B G_{2}^{T}$ to assign a self-conjugate set $\left\{\lambda_{j}\right\}_{j=1}^{k} \subset$ $\sigma(P)$ into $\left\{\mu_{j}\right\}_{j=1}^{k}$, while keeping the rest of $\sigma(P)$ unchanged.

There now exist several computationally viable methods for complete eigenvalue assignment (see [4] for details) in the standard firstorder state-space system. The robustness issue for complete assignment also has been dealt in the well-known papers [16] and [25]. Furthermore, robustness and minimum-feedback issues, either jointly or separately, were discussed in [1], [2], [18], [19], [26], and [27]. A few specialized methods for a more important practical variation of the problem, namely, the partial eigenvalue assignment problem, have feedback matrices, where $1 \leq b \leq n$. Without loss of generality, we 
also been proposed [24], [10], [27], [21], and the robustness issue for the first-order problem was discussed in the last two papers.

Because of practical computational and engineering difficulties including the increase in dimensionality, loss of explicable structures such as the sparsity, symmetry, etc., it is not advisable to solve a secondorder control system problem via transformation into a first-order standard state-space form. In view of this, the methods that deal directly in quadratic settings have been developed in recent years, [5], [6], [9], including methods for robust quadratic complete eigenvalue assignment [3], [22] and a the robust quadratic partial assignment [23].

In the meantime, a partial pole assignment algorithm for the cubic pencil was proposed in [20] and the robustness and minimum norm issues for this pencil using rather elementary optimization techniques was given in [7].

In this note, we give another close-look into the cubic problem and propose a new algorithm for robustness alone. The process of minimization of the condition number of the closed-loop system consists of judicious selection of eigenvectors from a given subspace. This is achieved by using rank-two updates for assignment of complex eigenvalues occurring in conjugate pairs. The process gives rise to a minimization problem with nonlinear constraints. Since the latter is a difficult problem, we propose here a new idea which involves a leastsquares problem with linear constraints. This reformulated problem is not mathematically equivalent to the original one; it is only an approximation. However, this reformulated least-square problem is much easier to solve and our numerical experimental results show that the results are quite acceptable.

It is to be mentioned in his context that the recent method of Qian and $\mathrm{Xu}$ [23] for the robust quadratic problem is also based on eigenvector selections. While our method is for a cubic polynomial (and theirs is for a quadratic pencil), the other main difference between our method and theirs is that they do not address the minimization problem to assign complex conjugate eigenvalues. More precisely, their method is a version of rank-one update even for complex conjugate eigenvalues. Ours is truly a rank-two updating algorithm for complex conjugate eigenvalues. Furthermore, their method, as stated by the authors themselves, does not always converge.

\section{A Parametric Solution of the Partial Pole ASSIGNMENT PROBLEM}

Let $\left\{\lambda_{j}\right\}_{j=1}^{3 n}$ be the eigenvalues of $P(\lambda)$, i.e., $\operatorname{det}\left(P\left(\lambda_{j}\right)\right)=0$ for $1 \leq j \leq 3 n$. Assume that $(\Lambda, X)$ and $(\tilde{\Lambda}, \tilde{X})$ be two eigenmatrix pairs of $P$. That is, they satisfy

$$
P(\Lambda) X:=M X \Lambda^{3}+C X \Lambda^{2}+K X \Lambda+L X=0
$$

and

$$
P(\tilde{\Lambda}) \tilde{X}:=M \tilde{X} \tilde{\Lambda}^{3}+C \tilde{X} \tilde{\Lambda}^{2}+K \tilde{X} \tilde{\Lambda}+L \tilde{X}=0
$$

Suppose that

$$
\sigma(\Lambda)=\left\{\lambda_{j}\right\}_{j=1}^{k} \quad \text { and } \quad \sigma(\tilde{\Lambda})=\left\{\lambda_{j}\right\}_{j=k+1}^{3 n}
$$

where $1 \leq k<3 n$ and $\left\{\lambda_{j}\right\}_{j=1}^{k}$ and $\left\{\lambda_{j}\right\}_{j=k+1}^{3 n}$ are two self-conjugate sets. Now let the self-conjugate set $\left\{\lambda_{j}\right\}_{j=1}^{k}$ be arranged as: $\left\{\lambda_{j}\right\}_{j=1}^{k}=\left\{\left\{\lambda_{2 \ell-1}, \lambda_{2 \ell}\right\}_{\ell=1}^{m_{1}},\left\{\lambda_{j}\right\}_{j=2 m_{1}+1}^{k}\right\}$, where $0 \leq m_{1} \leq k / 2,\left\{\lambda_{2 \ell-1}, \lambda_{\ell}\right\}_{\ell=1}^{m_{1}}$ are pairs of conjugate complex numbers with nonzero imaginary parts, and $\left\{\lambda_{j}\right\}_{j=2 m_{1}+1}^{k}$ are all real numbers. We aim to assign $\left\{\lambda_{j}\right\}_{j=1}^{k}$ into a self-conjugate set of complex numbers $\left\{\mu_{j}\right\}_{j=1}^{k}$. For this purpose, we assume that $\left\{\mu_{j}\right\}_{j=1}^{k} \cap \sigma(P)=\emptyset$.

Likewise, let us partition $\left\{\mu_{j}\right\}_{j=1}^{k}=$ $\left\{\left\{\mu_{2 r-1}, \mu_{2 r}\right\}_{r=1}^{m_{2}},\left\{\mu_{j}\right\}_{j=2 m_{2}+1}^{k}\right\}$, where $0 \leq m_{2} \leq k / 2$, $\left\{\mu_{2 r-1}, \mu_{2 r}\right\}_{r=1}^{m_{2}}$ are pairs of conjugate complex numbers with nonzero imaginary parts, and $\left\{\mu_{j}\right\}_{j=2 m_{2}+1}^{k}$ are all real numbers. Correspondingly, the eigenvectors associated with $\left\{\lambda_{j}\right\}_{j=1}^{k}$ are grouped into

$$
\left\{\left\{x_{2 \ell-1}, x_{2 \ell}\right\}_{\ell=1}^{m_{1}},\left\{x_{j}\right\}_{j=2 m_{1}+1}^{k}\right\}
$$

where $x_{2 \ell-1}=\bar{x}_{2 \ell}$ for all $1 \leq \ell \leq m_{1}$ and $\left\{x_{j}\right\}_{j=2 m_{1}+1}^{k}$ are real vectors. Notice that $m_{1}$ is not necessarily equal to $m_{2}$. Now, suppose that $\mathcal{U}=\left[u_{1}, \ldots, u_{k}\right]$ is a $b \times k$ complex matrix with column vectors $u_{j}$ satisfying

$$
\begin{cases}u_{2 r-1}=\bar{u}_{2 r} & \text { for } 1 \leq r \leq m_{2} \\ u_{j} \in \mathbb{R}^{p \times 1} & \text { for } 2 m_{2}+1 \leq j \leq k\end{cases}
$$

Subsequently, let $z_{j}$ be the $j$ th column of $B \mathcal{U}$, i.e., $z_{j}=B u_{j} \neq 0$ for $1 \leq j \leq k$. Notice that $\left\{z_{j}\right\}_{j=1}^{k}$ satisfy the same relations as in (1). In view of the above, we define

$$
y_{j}=P\left(\mu_{j}\right)^{-1} z_{j}, \quad 1 \leq j \leq k .
$$

That is, $y_{j}$ satisfies

$P\left(\mu_{j}\right) y_{j}=M y_{j} \mu_{j}^{3}+C y_{j} \mu_{j}^{2}+K y_{j} \mu_{j}+L y_{j}=z_{j}, \quad 1 \leq j \leq k$.

Notice that $y_{j} \neq 0$ for all $1 \leq j \leq k$. It should be noted that the degrees of freedom in the choice of $y_{j}$ is reflected by the degrees of freedom in choosing $z_{j}$ for any given $\mu_{j}$. The following solution of the partial eigenvalue assignment for the cubic pencil (2) has been proposed in [7].

Theorem 2.1: Let $\sigma(\Lambda) \cap \sigma(\tilde{\Lambda})=\emptyset$ and let the gain matrices be chosen as

$$
\begin{aligned}
F= & M X \Lambda \xi \\
G_{1}= & {\left[-\omega\left(K_{1}+\rho K_{2}\right)+\rho K_{2}\right] X \xi+(1-\omega)\left[M X \Lambda^{2}\right.} \\
& \left.+\left(C_{1}+\rho C_{2}\right) X \Lambda\right] \xi \\
G_{2}= & \frac{1}{\rho}\left\{\left[\omega\left(K_{1}+\rho K_{2}\right)-\rho K_{2}\right] X \xi\right. \\
& \left.+\omega\left[M X \Lambda^{2}+\left(C_{1}+\rho C_{2}\right) X \Lambda\right] \xi\right\}
\end{aligned}
$$

where $\xi \in \mathbf{C}^{k \times b}$ is arbitrary. (i) Then $(\tilde{X}, \tilde{\Lambda})$ is an eigenmatrix pair of $P_{c}(\lambda)$, i.e., $P_{c}(\tilde{\Lambda}) \tilde{X}=0$. ii) Moreover, if $\xi$ is chosen so as to satisfy the system of $k \times k$ linear algebraic equations

$$
\xi^{T} \Psi=\mathcal{U}
$$

where $\Psi$ is a solution of the Sylvester equation

$$
\Psi \Gamma-\Lambda \Psi=\Lambda X^{T} B \mathcal{U} \quad \Gamma=\operatorname{diag}\left(\mu_{1}, \ldots, \mu_{k}\right)
$$

then $\sigma\left\{P_{c}(\lambda)\right\}=\left\{\mu_{1}, \ldots, \mu_{k} ; \lambda_{k+1} \ldots, \lambda_{3 n}\right\}$ and $y_{j}$ is an eigenvector of $P_{c}(\lambda)$ associated with $\mu_{j}$ for $j=1, \ldots, k$. 


\section{MEASURE OF RobUSTness}

In this section, we present a measure of the robustness of the eigenvalue problem for cubic pencils which will be used later on to measure the robustness of the partial pole assignment problem. One obvious way is to apply various available measures of the robustness to the first order linearization of the cubic pencil. However, this does not comply with the principle of the note. Therefore, we work directly on the cubic pencil. Let $A(\lambda)=A_{3} \lambda^{3}+A_{2} \lambda^{2}+A_{1} \lambda+A_{0}$ be a cubic pencil with $\operatorname{det} A_{3} \neq 0$. Assume that $(U, J, V)$ is a Jordan triple of $A(\lambda)$ with $J=\operatorname{diag}\left(\lambda_{1}, \ldots, \lambda_{3 n}\right)$, i.e., $(U, J)$ is a Jordan pair of $A(\lambda)$ and

$$
V=\left[\begin{array}{c}
U \\
U J \\
U J^{2}
\end{array}\right]^{-1}\left[\begin{array}{c}
0 \\
0 \\
A_{3}^{-1}
\end{array}\right]=\left[\begin{array}{c}
U \\
U J \\
A_{3} U J^{2}
\end{array}\right]^{-1}\left[\begin{array}{l}
0 \\
0 \\
I
\end{array}\right]
$$

Let $\mu \notin \sigma(A)$. Then the inverse of $A(\mu)$ is given by

$$
A^{-1}(\mu)=U(\mu I-J)^{-1} V
$$

Suppose that the cubic pencil $A(\lambda)$ is perturbed into $\tilde{A}(\lambda)$ with associated coefficient matrices $A_{l}$ being perturbed into $A_{l}+E_{l}$ for $0 \leq l \leq 3$. We now prove the following.

Leema 3.1: Let $\mu \in \sigma(\tilde{A})$ then

$$
\min _{j}\left|\mu-\lambda_{j}\right| \leq\|U\|\|V\| \sum_{l=0}^{3}\left\|E_{l}\right\||\mu|^{l}
$$

where $\|\cdot\|$ is any matrix norm.

Proof: If $\mu \in \sigma(A)$ then (8) is trivial. Thus, we assume that $\mu \notin \sigma(A)$ and $x$ is a nonzero vector satisfying $\tilde{A}(\mu) x=0$. It is clear that

$$
[A(\mu)-\tilde{A}(\mu)] x=A(\mu) x
$$

and so

$$
A^{-1}(\mu)[A(\mu)-\tilde{A}(\mu)] x=x .
$$

Hence, we have that

$$
1 \leq\left\|A^{-1}(\mu)[A(\mu)-\tilde{A}(\mu)]\right\|
$$

From (7) and the form of $J$, the bound (8) follows immediately.

In view of this lemma, we define the condition number for the eigenvalues of $A(\lambda)$ to be $\kappa(A)=\|U\|\|V\|$. For computational simplicity, we will use the Frobenius norm throughout the rest of the note. Now, we can discuss the condition number for the eigenvalues of the closed-loop pencil $P_{c}(\lambda)$. Let us define

$$
Y=\left[y_{1}, \ldots, y_{k}, x_{k+1}, \ldots, x_{3 n}\right]
$$

To illuminate the main idea, we assume that $(Y, D)$ is a Jordan pair of $P_{c}(\lambda)$. That is, $(Y, D, Z)$ with

$$
Z=\left[\begin{array}{c}
Y \\
Y D \\
M Y D^{2}
\end{array}\right]^{-1}\left[\begin{array}{l}
0 \\
0 \\
I
\end{array}\right]=(\tilde{M} S)^{-1}\left[\begin{array}{l}
0 \\
0 \\
I
\end{array}\right]
$$

form a Jordan triple of $P_{c}(\lambda)$, where $\tilde{M}=I \oplus M$ and

$$
S=\left[\begin{array}{c}
Y \\
Y D \\
Y D^{2}
\end{array}\right]
$$

Therefore, it follows that $\kappa\left(P_{c}\right)=\|Y\|\|Z\|$. It should be noted that for the partial eigenvalue assignment problem only the first $k$ column vectors in $Y$ can be freely chosen. In other words, the condition number $\kappa\left(P_{c}\right)$ is partially minimized. However, note that even though the gain matrices $F, G_{1}, G_{2}$ given in (5) leave the remaining eigenpairs $\left\{\left(\lambda_{j}, x_{j}\right)\right\}_{j=k+1}^{3 n}$ unchanged, the condition numbers for the remaining eigenvalues can still be perturbed. It is therefore reasonable to consider the global condition number $\kappa\left(P_{c}\right)$ even for the partial pole assignment problem.

\section{Minimization OF $\kappa\left(P_{c}\right)$}

As indicated in [3] it is difficult to select eigenvectors to directly minimize $\kappa\left(P_{c}\right)$. However, by normalizing all eigenvectors in $Y$, we can see that $\kappa\left(P_{c}\right)=\|Y\|\|Z\| \leq \sqrt{3 n^{2}}\left\|\tilde{M}^{-1}\right\|\left\|S^{-1}\right\|$. Thus, to achieve our goal, it suffices to minimize $\left\|S^{-1}\right\|$. We will use a rank-2 update technique proposed in [16] to minimize $\left\|S^{-1}\right\|$ by selecting appropriate eigenvectors. Since all complex column vectors of $S$ appear in complex-conjugate pairs, we can define a real matrix $\hat{S}$ as follows: if the $j$ th column vector of $S$ is real, then $\hat{S}$ has the same $j$ th column vector. If the $j$ th and $(j+1)$ th column vectors of $S$ form complex-conjugate pair, say $v$ and $\bar{v}$, then the $j$ th column of $\hat{S}$ is defined to be the real part of $v$ and the $(j+1)$ th column of $\hat{S}$ is the imaginary part of $v$. It is easy to check that $\hat{S}$ is nonsingular if and only if $S$ is nonsingular and $\left\|S^{-1}\right\| \leq \tilde{C}\left\|\hat{S}^{-1}\right\|$ for some constant $\tilde{C}>0$. Therefore, $\left\|\hat{S}^{-1}\right\|$ can be minimized by the column update technique.

Since the rank-2 update method gives rise to a minimization problem with nonlinear constraints, which needs to be solved by complicated numerical methods, we reformulate the problem into a least-squares problem with linear constraints. The assignment of the real eigenvalues can be handled by using a rank-1 update and has been discussed in more detail in [8].

Let $\mu=\mu_{r}+\imath \mu_{i}$ be a complex eigenvalue that needs to be assigned, and $y=y_{r}+\imath y_{i}$ with $y_{r}^{T} y_{r}+y_{i}^{T} y_{i}=1$ be the associated eigenvector, where $\imath=\sqrt{-1}$. Then rearranging $\hat{S}$ yields $\hat{S}=[v, V]$, where

$$
v=\left[\begin{array}{cc}
y_{r} & y_{i} \\
\mu_{r} y_{r}-\mu_{i} y_{i} & \mu_{r} y_{i}+\mu_{i} y_{r} \\
\left(\mu_{r}^{2}-\mu_{i}^{2}\right) y_{r}-2 \mu_{r} \mu_{i} y_{i} & \left(\mu_{r}^{2}-\mu_{i}^{2}\right) y_{i}+2 \mu_{r} \mu_{i} y_{r}
\end{array}\right]
$$

Let the QR decomposition of $V$ be

$$
V=\left[q_{V}, Q_{V}\right]\left[\begin{array}{c}
0^{T} \\
R_{V}
\end{array}\right]=\tilde{Q}_{V}\left[\begin{array}{c}
0^{T} \\
R_{V}
\end{array}\right] \quad \tilde{Q}_{V}=\left[q_{V}, Q_{V}\right]
$$


then

$$
\hat{S}=[v, V]=\tilde{Q}_{V}\left[\begin{array}{cc}
q_{V}^{T} v & 0^{T} \\
Q_{V}^{T} v & R_{V}
\end{array}\right]
$$

So the inverse of $\hat{S}$ is

$$
\hat{S}^{-1}=\left[\begin{array}{cc}
\gamma_{2}^{-1} & 0^{T} \\
-R_{V}^{-1} Q_{V}^{T} v \gamma_{2}^{-1} & R_{V}^{-1}
\end{array}\right]
$$

where $\gamma_{2}=q_{V}^{T} v$ is a $2 \times 2$ matrix. Thus, to minimize $\hat{S}^{-1}$ we must minimize

$$
\left\|\left[\begin{array}{c}
\gamma_{2}^{-1} \\
R_{V}^{-1} Q_{V}^{T} v \gamma_{2}^{-1}
\end{array}\right]\right\| .
$$

Let the QR decomposition of $P(\mu)^{-1} B$ be $P(\mu)^{-1} B=Q_{B} R_{B}$, where $Q_{B}$ satisfies $Q_{B}^{H} Q_{B}=I$ with $Q_{B}^{H}$ being the Hermitian of $Q_{B}$. Consequently, if we let $Q_{B}=Q_{B r}+\imath Q_{B i}$, then we have $Q_{B r}^{T} Q_{B r}+$ $Q_{B i}^{T} Q_{B i}=I$ and $Q_{B r}^{T} Q_{B i}-Q_{B i}^{T} Q_{B r}=0$. In view of the structure of $v$, we can set $v=\mathcal{B H}$, where

$\mathcal{B}=\left[\begin{array}{cl}Q_{B r} & Q_{B i} \\ \mu_{r} Q_{B r}-\mu_{i} Q_{B i} & \mu_{r} Q_{B i}+\mu_{i} Q_{B r} \\ \left(\mu_{r}^{2}-\mu_{i}^{2}\right) Q_{B r}-2 \mu_{r} \mu_{i} Q_{B i} & \left(\mu_{r}^{2}-\mu_{i}^{2}\right) Q_{B i}+2 \mu_{r} \mu_{i} Q_{B r}\end{array}\right]$ and

$\mathcal{H}=\left[\begin{array}{cc}h_{r} & h_{i} \\ -h_{i} & h_{r}\end{array}\right] \in \mathbb{R}^{2 b \times 2} \quad$ with $\quad h_{r}^{T} h_{r}+h_{i}^{T} h_{i}=1$.

Performing the $\mathrm{QR}$ decomposition on $q_{V}^{T} \mathcal{B}$ gives

$$
q_{V}^{T} \mathcal{B}=\left[\alpha_{2}, 0\right]\left[\begin{array}{c}
q_{2}^{T} \\
Q_{2}^{T}
\end{array}\right]=\alpha_{2} q_{2}^{T}
$$

Now, let us denote $\phi=q_{2}^{T} \mathcal{H} \quad \psi=Q_{2}^{T} \mathcal{H}$, and $\Phi^{T}=\left[\phi^{T}, \psi^{T}\right]$, then from $\mathcal{H}^{T} \mathcal{H}=I$ we have $\Phi^{T} \Phi=I$. Thus, we can find $\Psi$ such that $[\Phi, \Psi]$ is orthogonal. Also, it follows from (11) that $\gamma_{2}=\alpha_{2} q_{2}^{T} \mathcal{H}=$ $\alpha_{2} \phi$. Note that $\Psi^{T} \Phi=0$. Consequently, we obtain that

$$
\begin{aligned}
\left\|\gamma_{2}^{-1}\right\| & =\left\|\phi^{-1} \alpha_{2}^{-1}\right\|=\left\|\phi^{-1} \alpha_{2}^{-1}\right\|=\left\|[\Phi, \Psi]^{T} \Phi \phi^{-1} \alpha_{2}^{-1}\right\| \\
& \left.=\left\|\Phi \phi^{-1} \alpha_{2}^{-1}\right\|\right]=\left\|\psi \phi^{-1} \alpha_{2}^{-1}\right\|
\end{aligned}
$$

and

$$
\begin{aligned}
\left\|R_{V}^{-1} Q_{V}^{T} v \gamma_{2}^{-1}\right\| & =\left\|R_{V}^{-1} Q_{V}^{T} \mathcal{B}\left[q_{2}, Q_{2}\right] \Phi \gamma_{2}^{-1}\right\| \\
& =\left\|R_{V}^{-1} Q_{V}^{T} \mathcal{B}\left[q_{2}, Q_{2}\right] I \psi \phi^{-1} \alpha_{2}^{-1}\right\| .
\end{aligned}
$$

Therefore, we need to solve the minimization problem

$$
\begin{aligned}
\min _{\mathcal{H}}\left\|\mathbb{R}_{V}^{-1} Q_{V}^{T} \mathcal{B}\left[q_{2}, Q_{2}\right] \alpha_{2}^{-1} \psi \phi^{-1} \alpha_{2}^{-1}\right\| \\
\quad=\min _{\mathcal{H}}\left\|\left[q_{2}, Q_{2}\right]^{T} \mathbb{R}_{V}^{-1} Q_{V}^{T} \mathcal{B}\left(q_{2} \alpha_{2}^{-1}+Q_{2} \psi \phi^{-1} \alpha_{2}^{-1}\right)\right\|
\end{aligned}
$$

with $\mathcal{H} \in \mathbb{R}^{2 b \times 2}$ subject to constraints (10). One possible way to solve the minimization problem (12) is to regroup $\psi \phi^{-1} \alpha_{2}^{-1}$ as new variables, say $\psi \phi^{-1} \alpha_{2}^{-1}=\hat{\mathcal{H}}$, and then problem (12) becomes equivalent to

$$
\min \hat{\mathcal{H}} \min \left\|\left[\begin{array}{c}
\left.q_{2}, Q_{2}\right]^{T} \\
R_{V}^{-1} Q_{V}^{T} \mathcal{B}
\end{array}\right]\left(q_{2} \alpha_{2}^{-1}+Q_{2} \hat{\mathcal{H}}\right)\right\|
$$

with $\hat{\mathcal{H}}$ subject to some nonlinear constraints. However, the problem (13) is very difficult to handle numerically. Here we will take a different approach to deal with (13). We show how to reformulate the problem (13) into a least squares problem with linear constraints which can be solved easily by standard numerical methods. We first observe that

$$
\begin{array}{r}
\left\|\left[q_{2}, Q_{2}\right]^{T} \mathbb{R}_{V}^{-1} Q_{V}^{T} \mathcal{B}\left(q_{2} \alpha_{2}^{-1}+Q_{2} \psi \phi^{-1} \alpha_{2}^{-1}\right)\right\| \leq \|\left[q_{2}, Q_{2}\right]^{T} \mathbb{R}_{V}^{-1} \\
Q_{V}^{T} \mathcal{B}\|\| \alpha_{2}^{-1}\|\| q_{2}+Q_{2} \psi \phi^{-1} \| .
\end{array}
$$

Therefore, it suffices to minimize

$$
\left\|q_{2}+Q_{2} \psi \phi^{-1}\right\|=\left\|q_{2}+Q_{2} Q_{2}^{T} \mathcal{H}\left(q_{2}^{T} \mathcal{H}\right)^{-1}\right\|
$$

with $\mathcal{H}$ satisfying (10). Note that $Q_{2} Q_{2}^{T}(c \mathcal{H})\left(c q_{2}^{T} \mathcal{H}\right)^{-1}=$ $Q_{2} Q_{2}^{T} \mathcal{H}\left(q_{2}^{T} \mathcal{H}\right)^{-1}$ for any $0 \neq c \in \mathbb{R}$. Thus, the unit length constraint $h_{r}^{T} h_{r}+h_{i}^{T} h_{i}=1$ in (10) can be removed. Since the inverse of $q_{2}^{T} \mathcal{H}$ is involved in (14), instead of solving (14), it is reasonable to consider the following minimization problem:

$$
\left\{\begin{array}{c}
\min \mathcal{H}\left\|q_{2}+Q_{2} Q_{2}^{T} \mathcal{H}\right\| \\
\text { subject to } q_{2}^{T} \mathcal{H}=I
\end{array}\right.
$$

Now, let

$$
\tilde{h}=\left[\begin{array}{l}
h_{r} \\
h_{i}
\end{array}\right] \in \mathbb{R}^{2 b \times 1} \quad \text { and } \quad q_{2}=\left[\begin{array}{ll}
q_{11} & q_{12} \\
q_{21} & q_{22}
\end{array}\right] \in \mathbb{R}^{2 b \times 2}
$$

then the constraint $q_{2}^{T} \mathcal{H}=I$ becomes

$$
\left[\begin{array}{cc}
q_{11}^{T} & -q_{21}^{T} \\
q_{21}^{T} & q_{11}^{T} \\
q_{12}^{T} & -q_{22}^{T} \\
q_{22}^{T} & q_{12}^{T}
\end{array}\right] \tilde{h}=\left[\begin{array}{l}
1 \\
0 \\
0 \\
1
\end{array}\right]
$$

which gives to an affine hyperplane whenever $b \geq 3$. Next, we denote $Q_{2} Q_{2}^{T}=\left[p_{1}, p_{2}\right]$, where $p_{1}, p_{2} \in \mathbb{R}^{2 b \times b}$. Thus, the minimization problem (15) is equivalent to

$$
\left\{\begin{array}{l}
\min _{\tilde{h}}\left\|\left[\begin{array}{l}
q_{11} \\
q_{21}
\end{array}\right]+\left[p_{1},-p_{2}\right] \tilde{h}\right\|^{2}+\left\|\left[\begin{array}{l}
q_{12} \\
q_{22}
\end{array}\right]+\left[p_{2}, p_{1}\right] \tilde{h}\right\|^{2} \\
\text { subject to }(16)
\end{array}\right.
$$

It should be noted that the objective function in (17) can be transformed into $\|c+N \tilde{h}\|^{2}+$ constant for some $c \in \mathbb{R}^{2 b \times 1}$, where $N^{T} N$ is the Cholesky factorization of $\left[p_{1},-p_{2}\right]^{T}\left[p_{1},-p_{2}\right]+\left[p_{2}, p_{1}\right]^{T}\left[p_{2}, p_{1}\right]$. Therefore, to solve (17), it suffices to consider the following least squares problem with linear equality constraints:

$$
\left\{\begin{array}{c}
\min _{\tilde{h}}\|c+N \tilde{h}\|^{2} \\
\text { subject to }(16)
\end{array}\right.
$$

which can be easily solved numerically [13]. Let $\tilde{h}_{\text {min }}$ be the minimizer of (18), then the $j$ th and $j+1$ th columns of $\mathcal{U}$ are $u$ and $\bar{u}$ with $u=$ $R_{B}^{-1}\left(h_{1}+\imath h_{2}\right)$, and $h_{1}$ and $h_{2}$ are defined by $\left(\tilde{h}_{\min }\right) /\left(\left\|\tilde{h}_{\min }\right\|\right)=$ $\left[\begin{array}{l}h_{1} \\ h_{2}\end{array}\right]$. 


\section{Algorithm 5.1 a COMPutational Algorithm FOR Robust PARTIAl EIgENVAlue AssignMENT In CUBIC PENCILS}

In this section, we give a numerical scheme to solve the robust partial pole assignment problem for the cubic pencil $P(\lambda)$.

Step 1: Find the QR decomposition of $P\left(\mu_{j}\right)^{-1} B$ :

$$
P\left(\mu_{j}\right)^{-1} B=Q_{B, j} R_{B, j}, \quad 1 \leq j \leq k
$$

where $Q_{B, 2 r-1}=\bar{Q}_{B, 2 r}$. Take $\mathcal{H}=\left[h_{1}, h_{2}, \ldots, h_{k}\right] \in C^{b \times k}$ with unit column vectors such that the relations (10) are satisfied. Set $y_{j}=$ $Q_{B, j} h_{j}$ for $1 \leq j \leq k$ and form

$$
S=\left[\begin{array}{c}
Y \\
Y D \\
Y D^{2}
\end{array}\right]
$$

where

$$
Y=\left[y_{1}, \ldots, y_{k}, x_{k+1}, \ldots, x_{3 n}\right]
$$

and

$$
D=\operatorname{diag}\left(\mu_{1}, \ldots, \mu_{k}, \lambda_{k+1}, \ldots, \lambda_{3 n}\right) .
$$

From $S$ construct a new matrix $\hat{S}$ with real column vectors as in Section IV.

Step 2.: Minimize $\kappa\left(P_{c}\right)$ by performing the minimization process described in Subsection IV.

Remark: The minimization process is stopped in at most $k$ sweeps. At the end, we obtain a matrix $\mathcal{U} \in C^{\lfloor\S \|}$.

Step 3: Find the feedback matrices $F, G_{1}$, and $G_{2}$ from the formulas in (5) with $\xi$ determined from (6).

\section{A. Structure Preserving Properties of Algorithm 5.1}

Advantages of the special structures of the matrices $M, C$, and $K$, often offered by practical problems, can be taken in implementing the algorithm, as described in the following.

- Forming the matrix $P\left(\mu_{j}\right)^{-1} B$ in Step 1 is computationally equivalent to solving linear systems of algebraic equations: $P\left(\mu_{j}\right) X=B$, where the matrix $X$ needs to be determined. The special structures of $M, C, K$, and $L$, can be exploited in both forming $P\left(\mu_{j}\right)$ and solving the linear systems. For example, in many practical instances, the matrix $M$ is diagonal, $K$ is symmetric tridiagonal and $L$ and $C$ are small matrices. If this is the case, the matrix $P\left(\mu_{j}\right)$ for a given $\mu_{j}$ is almost a tridiagonal matrix and solving a tridiagonal system requires $O(n)$ flops compared to $O\left(n^{3}\right)$ flops required to solve an arbitrary system [11].

- Special structures of these matrices can be exploited in computing the QR factorization in Step 1 and the subsequent solution of the least-squares problem in Step 2. (Note that the QR factorization of a tridiagonal matrix requires $O(n)$ flops compared to $O\left(n^{3}\right)$ flops for an arbitray matrix) [11].

- The matrix multiplications in Step 3 in forming the matrices $F$, $G 1$, and $G 2$ using (5) can also be efficiently performed using the structures of those matrices. Furthermore, since computations here are independent and rich is matrix multiplications, these computations are ideal for high-performance computing, which is an additional advantage.

Unfortunately such computational advantages are not possible if the problem had been solved by transforming to a standard state-space form; all the exploitable properties, such as the sparsity, positive definiteness, and others would be completely destroyed by such a transformation.
TABLE I

Initial Relative Errors of the Assigned Eigenvalues

\begin{tabular}{|c|c|}
\hline$j$ & $\left|\mu_{j}-\hat{\mu}_{j}\right|$ \\
\hline 1 & $\frac{\left|\mu_{j}\right|}{2.740328429155475 e-10}$ \\
\hline 3 & $3.153920390187170 e-10$ \\
\hline 5 & $3.259099552478408 e-07$ \\
\hline 7 & $3.879586188962258 e-07$ \\
\hline 9 & $5.032058840640367 e-08$ \\
\hline 11 & $2.385611827580338 e-08$ \\
\hline
\end{tabular}

$$
\begin{aligned}
& \begin{array}{|c|c|}
\hline j & \frac{\left|\mu_{j}-\hat{\mu}_{j}\right|}{\left|\mu_{j}\right|} \\
\hline 1 & 5.727221205072635 e-06 \\
3 & 5.753274206437847 e-06 \\
5 & 8.374374072298398 e-05 \\
7 & 8.379728107210083 e-05 \\
9 & 1.181655907299993 e-05 \\
11 & 1.174263884686994 e-05 \\
\hline
\end{array} \\
& \max \left\{\frac{\left|\lambda_{j}-\hat{\lambda}_{j}\right|}{\left|\lambda_{j}\right|}, j=13, \ldots, 126\right\}=3.401523930 e-02 \text {. }
\end{aligned}
$$

TABLE II

Final Relative Errors of Assigned Eigenvalues

Numerical Experiment: A numerical experiment was performed with simulated data obtained from the Boeing Company. The sizes of matrices $M, C_{1}, C_{2}, K_{1}$. and $K_{2}$ are all $42 \times 42$. Therefore, the total number of eigenvalues (counting multiplicity) is 126 .

A set of six pairs of complex eigenvalues $\left\{\lambda_{j}\right\}_{j=1}^{12}=$ $\left\{\lambda_{1}, \lambda_{2}, \lambda_{3}, \lambda_{4}, \ldots, \lambda_{11}, \lambda_{12}\right\}=\left\{\lambda_{1}, \bar{\lambda}_{1}, \lambda_{3}, \bar{\lambda}_{3}, \ldots, \lambda_{11}, \bar{\lambda}_{11}\right\}$ were assigned to the eigenvalues

$$
\begin{aligned}
\left\{\mu_{j}\right\}_{j=1}^{12} & =\left\{\mu_{1}, \mu_{2}, \mu_{3}, \mu_{4}, \ldots, \mu_{11}, \mu_{12}\right\} \\
& =\left\{\mu_{1}, \bar{\mu}_{1}, \mu_{3}, \bar{\mu}_{3}, \ldots, \mu_{11}, \bar{\mu}_{11}\right\}
\end{aligned}
$$

Their specific values are omitted for the lack of space. We choose the input matrix $B=\left[b_{1}, b_{2}, b_{3}, b_{4}\right]$ with $b_{1}^{T}=(1 / \sqrt{21})[1,0,-1,0,1,0,-1,0, \ldots,-1,0,1], b_{2}^{T}=b_{1}^{T}$ except $b_{2}(21)=0.99, b_{3}^{T}=(1 / \sqrt{42})[1,1, \ldots, 1]$ and $b_{4}^{T}=b_{3}^{T}$ except $b_{4}(42)=1.03$.

The initial relative errors (without using Algorithm 5.1) and the Final relative errors (with the use of Algorithm 5.1) are plotted in Tables I and II. Six sweeps were required to move the eigenvalues.

The results show an improvement of both the assigned and the remaining eigenvalues with the use of Algorithm 5.1, as expected.

The results obtained in an earlier paper [7] were a little bit more accurate than those presented in the above Tables. This is because in [7], minimizations of both feedback norms and eigenvector conditioning were considered and, furthermore, a more powerful optimization technique was used there rather than the simple least-squares technique used in the current note. Note that the technique in [7] is quite computationally intensive, it involves solution of a nonlinear unconstrained optimization problem which is to be solved iteratively and requires evaluation of the first derivative of the objective function to find the search direction at each iteration, resulting in a very high-order of computational complexity and convergence problems. On the other hand, the technique of the present note requires solution of a least-squares problem which can be done in a finite number of steps using a direct method, such as the QR Factorization. Thus, the technique of this note is more efficient but is likely less accurate.

\section{CONCLUSION}

Numerically robust feedback control design concerns with choosing a suitable feedback matrix (matrices) such that the closed-loop eigenvalues are as insensitive as possible to small perturbations of the data. 
While several algorithms have been developed for computing robust feedback matrices for complete pole assignment in standard first-order state-space and also for the matrix second-order systems, such algorithms for partial pole-placement (which is more practical for large and sparse systems) are rare. In this note, a computationally simple least-squares based algorithm for robust partial pole placement in a cubic matrix pencil arising from modelling of vibrating structures with aerodynamics effects is proposed. It should be emphasized that here we formulate the rank-two update method (for complex poles) into a least square problem with linear constraints which can be solved easily by elementary linear algebra techniques. Moreover, our new algorithm is not an iterative method. For instance, if we want to assign $k$ pairs of complex poles, i.e., $2 \mathrm{k}$ number of eigenvalues then we only need to do exact $k$ steps in our method. Clearly, this new algorithm is much simpler and more efficient than the only other algorithm proposed so far for this problem.

\section{REFERENCES}

[1] S. P. Bhattacharyya and E. deSouza, "Pole assignment via Sylvester's equation," Syst. Control Lett., no. 4, pp. 261-263, 1982.

[2] R. Byers and S. G. Nash, "Approaches to robust pole assignment," Int. J. Control, no. 49, pp. 97-117, 1989.

[3] E. K. Chu and B. N. Datta, "Numerical robust pole assignment for second-order systems," Int. J. Control, no. 64, pp. 1113-1127, 1996.

[4] B. N. Datta, Numerical Methods for Linear Control Systems Design and Analysis. New York: Elsevier, 2003.

[5] B. N. Datta, S. Elhay, and Y. M. Ram, "Orthogonality and partial pole assignment for the symmetric definite quadratic pencil," Linear Alg. Appl., no. 257, pp. 29-48, 1997.

[6] B. N. Datta, S. Elhay, Y. M. Ram, and D. R. Sarkissian, "partial eigenstructure assignment for the quadratic pencil," J. Sound Vibrat., vol. 230, pp. 101-110, 2000.

[7] B. Datta, W.-W. Lin, and J.-N. Wang, "Robust and minimum gain partial pole assignment for a third order system," in Proc. IEEE Conf. Dec. Control, 2003, pp. 2358-2363.

[8] B. Datta, W.-W. Lin, and J.-N. Wang, "Robust partial pole assignment for vibrating systems with aerodynamic effects," in Proc. IEEE Conf. Dec. Control, 2004.

[9] B. N. Datta and D. R. Sarkissian, "Theory and computation of some inverse problems," Contemp. Math., vol. 280, pp. 221-240, 2001.

[10] B. N. Datta and D. R. Sarkissian, "Partial eigenvalue assignment in linear systems; Existence, uniqueness and numerical methods," in Proc. Math. Theory Networks Syst., 2002.

[11] B. N. Datta, Numerical Linear Algebra and Applications. CA: Brooks/Cole, 2005.

[12] W. R. Ferng, W. W. Lin, D. J. Pierce, and C.-S. Wang, "Nonequivalence transformation of lambda-matrix eigenproblems and model embedding approach to model tuning," Numer. Lin. Alg. Appl., vol. 8, pp. 53-70, 2001.

[13] G. H. Golub and C. F. Van Laon, Matrix Computations, 3rd ed. Baltimore, MD: Johns Hopkins Univ. Press, 1996.

[14] F. Hoblit, Gust Loads on Aircraft: Concepts and Applications 1988, AIAA Education Series.

[15] D. Inman, Vibration with Control, Measurement, and Stability. Englewood Cliffs, NJ: Prentice-Hall, 1989.

[16] J. Kautsky, N. K. Nichols, and P. Van Dooren, "Robust pole assignment in linear state feedback," Int. J. Control, vol. 41, pp. 1129-1155, 1985.

[17] J. Kautsky and N. K. Nichols, "Robust multiple eigenvalue assignment by state feedback in linear systems," in Linear Algebra and Its Role in Systems Theory. Providence, RI: AMS, 1985, pp. 253-264.

[18] L. H. Keel, J. A. Fleming, and S. P. Bhattacharyya, "Minimum norm pole assignment via Sylvester's equation," Contemp. Math., vol. 47, pp. 265-272, 1985.

[19] R. K. Kevin and S. P. Bhattacharyya, "Robust and well conditioned eigenstructure assignment via Sylvester's equation," Optm. Control Appl. Meth., vol. 4, pp. 205-212, 1983.

[20] W.-W. Lin and J.-N. Wang, "Partial pole assignment for the vibrating system with aerodynamic effect," Numer. Linear Algebra Appl., vol. 11, pp. 41-58, 2004.

[21] N. K. Nichols, "Robustness in partial pole placement," IEEE Trans. Autom. Control, vol. AC-32, no. 8, pp. 728-732, Aug. 1987.
[22] N. K. Nichols and J. Kautsky, "Robust eigenstructure assignment in quadratic matrix polynomials: Nonsingular case," SIAM J. Matrix Anal. Appl., vol. 23, pp. 77-102, 2001.

[23] J. Qian and S. Xu, "Robust partial eigenvalue assignment problem for the second-order system," J. Sound Vibrat., vol. 282, pp. 937-948, 2005.

[24] Y. Saad, "Projection and deflation methods for partial pole assignment in linear state feedback," IEEE Trans. Autom. Control, vol. 33, no. 2, pp. 290-297, Feb. 1988.

[25] A. L. Tits and Y. Yang, "Globally convergent algorithms for robust pole assignment by state feedback," IEEE Trans. Autom. Control, vol. 41, no. 10, pp. 1432-1452, Oct. 1996.

[26] A. Varga, "Robust and minimum norm pole assignment with periodic state feedback," in Proc. IEEE Conf. Dec. Control, 1998, pp. $3837-3842$.

[27] A. Varga, "Robust pole assignment techniques via state feedback," in Proc. IEEE Conf. Dec. Control, 2000, pp. 4655-4660.

[28] J.-N. Wang, S.-H. Chou, Y.-C. Chen, and W.-W. Lin, "Pole assignment for a vibrating system with aerodynamic effect," SIAM J. Control Optim., pp. 2116-2129, 2004.

\section{Analysis and Synthesis of Robust Control Systems Using Linear Parameter Dependent Lyapunov Functions}

\author{
José C. Geromel and Rubens H. Korogui
}

\begin{abstract}
This note provides sufficient robust stability conditions for continuous time polytopic systems. They are obtained from the Frobenius-Perron Theorem applied to the time derivative of a linear parameter dependent Lyapunov function and are expressed in terms of linear matrix inequalities (LMI). They contain as special cases, various sufficient stability conditions available in the literature to date. As a natural generalization, the determination of a guaranteed $\mathrm{H}_{2}$ cost is addressed. A new gain parametrization is introduced in order to make possible the state feedback robust control synthesis using parameter dependent Lyapunov functions through linear matrix inequalities. Numerical examples are included for illustration.
\end{abstract}

Index Terms-Linear matrix inequalities (LMIs), linear systems, robust control design, robust stability and performance.

\section{INTRODUCTION}

Robust stability and performance of polytopic systems has received special attention during the last decades. The main motivation for its development was the possibility to analyze and to design control strategies to cope with uncertain parameters, that is, parameters that can not be considered precisely known. Classes of linear systems denominated affine and polytopic came to light in order to put in evidence the linear dependence of the model with respect to the uncertain parameters. The first stability criterion denominated quadratic stability condition was established using a quadratic in the state Lyapunov function and independent of the unknown parameters, see [3] for a discussion on this point. Naturally, this condition provides conservative results but it is

Manuscript received May 25, 2005; revised November 14, 2005 and April 18, 2006. Recommended by Associate Editor A. Garulli. This work was supported by "Conselho Nacional de Desenvolvimento Científico e Tecnológico (CNPq)" and "Fundação de Amparo à Pesquisa do Estado de São Paulo (FAPESP)," Brazil.

The authors are with DSCE/School of Electrical and Computer Engineering, UNICAMP, 13083-970 Campinas, SP, Brazil (e-mail: geromel@dsce.fee.unicamp.br).

Digital Object Identifier 10.1109/TAC.2006.884958 